\title{
Stop-signal inhibition disrupted by damage to right inferior frontal gyrus in humans
}

\author{
Adam R Aron, Paul C Fletcher, Ed T Bullmore, Barbara J Sahakian \& Trevor W Robbins \\ Nat. Neurosci. 6, 115-116 (2003)
}

In the version of this article initially published online, the legend to Supplementary Figure 1 was omitted. The legend has now been appended to the online supplementary figure.

\begin{abstract}
Supplementary Figure 1 Data from left frontal patients. Correlations between stop signal reaction times (SSRT, ms) and the volume of damage to each region (SFG, IFG, MFG, ORB and MED, $\mathrm{cm}^{3}$ ) for each patient. SSRT for left frontal patients was $194 \pm 44.1 \mathrm{~ms}$, indicating intact response inhibition overall; confirmed by the fact that SSRT was significantly faster than for right frontals $(t=2.2, P<0.05)$. SSRT was not significantly positively correlated with damage to any of: SFG $(n=15, r=0.1$, n.s. $)$, MFG $(r=-0.47$, n.s. $)$, IFG $(r=-0.58, P=0.023)$, ORB $(P=0.3$, n.s. $)$ and MED $(r=0.3$, n.s. $)$.
\end{abstract}

\section{NF-kB functions in synaptic signaling and behavior}

\author{
Mollie K Meffert, Jolene M Chang, Brian J Wiltgen, Michael S Fanselow \& David Baltimore \\ Nat. Neurosci. 6, 1072-1078 (2003)
}

Due to a typesetting error in the Fig. 3 legend, the symbol $\mu$ was mistakenly printed as the letter ' $m$ '. This led to erroneous concentration

descriptions for reagents in panels $\mathbf{c}$ and $\mathbf{d}$. The corrected legend appears below.

Figure $3 \mathrm{Ca}^{2+}$-dependent pathway of NF-kB activation. (c,d) Cultures were unstimulated or stimulated with bicuculline $(50 \mu \mathrm{M},+4$-aminopyridine $5 \mu \mathrm{M})$, in the presence or absence of intracellular $\mathrm{Ca}^{2+}$ chelators (EGTA, BAPTA or $\mathrm{Br}_{2}-\mathrm{BAPTA}$ ), and subjected to EMSA or reporter assay. All cultures were preincubated in activity-inhibitors, which were washed out before loading with intracellular $\mathrm{Ca}^{2+}$ buffers and stimulation. (c) Averaged data from five separate EMSA experiments; error bars represent one s.e.m. $\mathrm{Br}_{2}$-BAPTA $(50 \mu \mathrm{M})$ did not significantly attenuate NF- $\mathrm{kB}$ activation compared to bicuculline

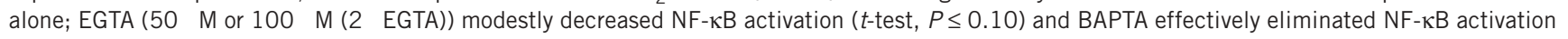
$(P \leq 0.001)$. (d) Cultures were co-infected with an NF- $\kappa B$-reporter gene ( $\kappa \mathrm{B}-/ u c)$ or an NFAT-reporter gene (NFAT-luc) and, to permit normalization, a constitutively expressed $\beta$-galactosidase. Data shown are averaged replicates from four separate assays. $\mathrm{Br}_{2}$-BAPTA (50 $\mu$ M) did not significantly attenuate NF- $\mathrm{KB}$ activation compared to bicuculline alone. EGTA (50 $\mu \mathrm{M}$ or $100 \mu \mathrm{M}(2 \times \mathrm{EGTA}))$ marginally, but not significantly $(P=0.3327)$, decreased $\kappa B-l u c i f e r a s e ~ a c t i v i t y$, and BAPTA effectively eliminated NF-KB transcriptional activation $(P \leq 0.001)$. Transcriptional activation from the NFAT-responsive element was significantly inhibited by either BAPTA or EGTA ( $P \leq 0.005$ for both).

\section{Expert face processing requires visual input to the right hemisphere during infancy}

Richard Le Grand, Catherine J Mondloch, Daphne Maurer \& Henry P Brent

Nat. Neurosci. 6, 1108-1112 (2003)

Due to a copy editing error, an extra symbol ('RE group') appeared in the key to Fig. 3. The corrected graph is shown below.

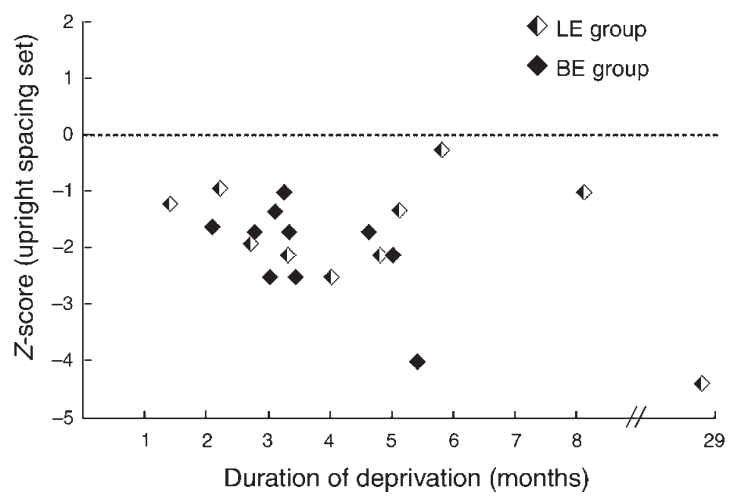

Figure 3 The effect of duration of visual deprivation on second-order relational processing. Individual Z-scores for accuracy on the upright spacing set for patients with deprivation affecting mainly the right hemisphere (LE group) are plotted as a function of the duration of visual deprivation from birth. For comparison, Z-scores are shown for patients $(n=10)$ with deprivation affecting both hemispheres (BE group). Negative scores represent deficits in units of standard deviation from the norm for the patient's age. 